\title{
A New Method For Machinery Fault Diagnoses Based On an Optimal Multiscale Morphological Filter
}

\author{
Wei Tan $1,2,{ }^{*}-$ Xiaoan Chen $^{1}$ - Shaojiang Dong 1 \\ ${ }^{1}$ State Key Laboratory of Mechanical Transmission, Chongqing University, China \\ 2 Key Laboratory of Advanced Manufacturing Technology for Automobile Parts, Chongqing University of Technology, China \\ In order to effectively eliminate the noise and extract the impulse components in the vibration signals, a new method based on an optimal \\ multiscale morphological filter is proposed. In this method, firstly, the average of the closing and opening operator is used to construct the \\ morphological filter, then the multiscale morphological filters' structure elements (SEs) are optimized and selected using a particle swarm \\ optimization algorithm (PSO). The noise in the original signal is filtered by the multiscale morphological filter. The proposed method was \\ evaluated by simulated signals and bearing fault signals. The results show that the method can effectively filter the noise and extract the \\ impulse characteristics of the vibration signals, which demonstrate the effectiveness of the proposed method.
}

Keywords: multiscale morphological filter, structure element, particle swarm optimization algorithm, noise reduction

\section{INTRODUCTION}

Rolling bearings are widely used in rotating machinery. Unexpected bearing failures can cause unscheduled downtime and loss. Therefore, it is very important to diagnose the fault in the bearing. However, even useful features in the bearing fault signals are often filled with strong background noise, which prevents the detection of bearing faults, so an effective signal processing method is desired to provide more information about the fault.

Recently, the mathematical morphological filter has been introduced into the fault diagnosis of machinery [1]. It can decompose the original signal into several physical parts according to certain geometric characteristics, thus overcoming the drawbacks of other signal processing methods such as the Fast Fourier transform (FFT) method, which cannot be used to the nonlinear and non stationary signal, the wavelet transform method, which has to choose the basic wavelet and needs a long time to compute [2], and the empirical mode decomposition (EMD) method, which has the problem of mode mixing [3].

There are some studies of morphology analysis in one-dimensional (1-D) signals; a flat structuring element (SE) with a length around 0.6 times the pulse repetition period was used to demodulate the fault signals [1]. An open-closing and close-opening combined morphological filter to de-noise the vibration signal of rotating machinery was proposed [4]. Zhang, et al. [5] introduced an approach based on the morphological filter to extract the features of the signal from a faulty gear according to the signal characteristics. Li and Xiao [6] introduced pattern classification based on a 1-D adaptive rank-order morphological filter. However, those studies required prior knowledge of the signals and a fixed SE in single-scale morphology analysis.

For a special signal, the characteristic features may be presented in multiscales. In order to extract these features, a multiscale morphology analysis is required. Multiscale morphology analysis can be used to extract morphological features of different scales and it is independent of prior knowledge when selecting SEs. Hence, it is often more feasible to remove the noise using a multiscale morphological filter [7].

The rest of this paper is organized as follows. In Section 1, the theory behind the morphological filter is introduced, and a multiscale morphological filter is constructed; the SEs are optimized by PSO. Sections 2 discuss the presented method in detail. Section 3 applies the proposed method to the simulated signal and Section 4 applies the proposed method to the bearing fault experiment. Some conclusions are given in Section 5.

\section{MORPHOLOGICAL FILTER ANALYSIS}

\subsection{The Fundamental Theory of Morphological Filter}

The morphological filter requires less computational time than other traditional signal processing methods. By constantly moving the SE to match the signal, feature extracting and de-noising can be achieved. For a signal $x(n)$ defined by $D_{f}=0,1, \ldots, N-1$ boundary, the $\operatorname{SE~} g(n)$ is a discrete function defined by $D_{g}=0,1, \ldots, M-1$ boundary, where $N \geq M$. The 
transformation consists of four basic operations: erosion, dilation, opening and closing:

$$
\begin{gathered}
(x \oplus g)(n)=\max [x(n-m)+g(n)], \\
(x \otimes g)(n)=\max [x(n+m)-g(n)], \\
(x \circ g)(n)=(x \otimes g \oplus g)(n), \\
(x \bullet g)(n)=(x \oplus g \otimes g)(n) .
\end{gathered}
$$

In the formula $\oplus, \otimes, \circ$ and $\bullet$ symbols corresponds to the dilation, erosion, opening and closing operation.

\subsection{Design of the Multiscale Morphology Filter}

Multiscale morphological filters were first presented by Maragos [7]. In this paper, we defined $T$ to denote the morphological operator as proposed by Maragos. Based on multiscale analysis, we can define:

$$
T_{\lambda}(x)=\lambda T(x / \lambda),
$$

where $\left\{T_{\lambda} \mid \lambda>0, \lambda \in N\right\}$.

Similarly, multiscale erosion and dilation can be defined as:

$$
\begin{aligned}
& (x \otimes g)_{\lambda}=\lambda[(x / \lambda) \otimes g]=x \otimes \lambda g, \\
& (x \oplus g)_{\lambda}=\lambda[(x / \lambda) \oplus g]=x \oplus \lambda g,
\end{aligned}
$$

where $\lambda g=g \oplus g \oplus \ldots \oplus g(\lambda-1)$ times.

In morphological operations, opening and closing operations have different processing performances. An opening operation could restrain the positive impulse and keep the negative impulse, while the closing operation will have inverse function. So, in practical applications, the relative morphological operation should be selected to correspond to the processing aim. However, it is sometimes it is difficult to get transcendental knowledge of practical positive and negative impulses; the general situation is that the positive and negative impulses are contained in practical data at the same time. Therefore, it is necessary to construct a morphological filtering algorithm with a combination of open and closed operations. In this article, the nonlinear filter was constructed in the form:

$$
y(x)=1 / 2[x \bullet g+x \circ g],
$$

where $y(x)$ is the filtered signal. So the nonlinear filter can be expressed as:

$$
\begin{aligned}
y_{\lambda}(x) & =\lambda y(x / \lambda)=\lambda / 2[(x / \lambda) \bullet g+(x / \lambda) \circ g]= \\
& =\lambda / 2[(x / \lambda) \oplus g \otimes g+(x / \lambda) \otimes g \oplus g]= \\
& =\lambda / 2[x \oplus \lambda g \otimes \lambda g+x \otimes \lambda g \oplus \lambda g],
\end{aligned}
$$

where the $\lambda$ is the scale parameter. If $\lambda=1$, the size of $g$ was set to $3 \times 3$. If $\lambda=2$, the size of $2 g$ was set to $5 \times 5$. So, the size of the $i g$ was set to $(2 i+1) \times(2 i+1)$ which according to the length of the noise period [8].

Because the triangle has good stability and good symmetry, the triangular shape SE was selected as an effective filtering window in this paper. The amplitudes of the SEs were optimized through PSO in this paper.

\subsection{Optimization of the Morphological Filter}

The PSO was chosen to optimize the SE of the morphological filter through the following formula [9]:

$$
\begin{gathered}
v_{i j}(t+1)=w v_{i j}(t)+c_{1} r_{1 j}\left(p_{i j}(t)-x_{i j}(t)\right)+ \\
+c_{2} r_{2 j}\left(p_{g_{j}}(t)-x_{i j}(t)\right), \\
x_{i j}(t+1)=x_{i j}(t)+v_{i j}(t+1),
\end{gathered}
$$

where the subscript $i$ represents the $i^{\text {th }}$ particle. $j$ represents the $j$-dimensional. The subscript $t$ represents the $t$ generation. $v_{i j}(t)$ is the velocity of the $i^{\text {th }}$ particle in the $t^{\text {th }}$ iteration; $x_{i j}(t)$ is the position of the $i^{\text {th }}$ particle; $p_{i j}(t)$ is the pbest position of the $i^{\text {th }}$ particle; $p_{g_{j}}$ is the gbest position (pbest represents the local optimum of the particles, gbest represents the overall situation optimum of the particles); $w$ represents the inertia weight. $c_{1}, c_{2}$ are learning factors. $r_{1} \sim U(0,1)$, $r_{2} \sim U(0,1)$ represent two independent random functions.

\subsection{Determination of the Fitness Function}

The signal-to-noise ratio (SNR) is selected as the fitness function:

$$
R_{S N}(x)=10 \log \frac{p(w)}{N(w)}, \quad p(w)=|Y(w)|^{2},
$$

where $R_{S N}(x)$ is the $S N R$ function of the system, $y(x)$ is the output of the filter, $Y(w)$ is the power spectrum of the output signal; $N(w)$ is the power spectrum of the noise that is removed from the original signal when the terminal interaction time is reached, to obtain the SNR value. 
The process of optimizing the SE based on the PSO is given below:

1. At the beginning of the optimization process, randomly initialize positions and velocities, pbest and gbest of the particles;

2. Set the parameters of SEs equal to the parameters of the particles' positions;

3. Use the SEs to construct the morphological filters;

4. Use the morphological filters to deal with the signal and get the filtered signal $y(x)$, then calculate the current fitness value $R\left(x_{i}\right)$ of each particle using Eq. (12);

5. Use the pbest to construct the morphological filter and calculate the fitness value $R\left(x_{p}\right)$, then compare the $R\left(x_{p}\right)$ with $R\left(x_{i}\right)$. If $R\left(x_{i}\right)$ is greater than $R\left(x_{p}\right)$, then set the parameters of particle $x_{i}$ to the pbest;

6. Use the gbest to construct the morphological filter and calculate the fitness value $R\left(x_{g}\right)$ and compare the $R\left(x_{g}\right)$ with $R\left(x_{g}\right)$. If $R\left(x_{p}\right)$ is greater than $R\left(x_{g}\right)$, then set the parameters of pbest to the gbest;

7. For each particle $i$ in the swarm, calculate positions $x_{i+1}$, velocities $v_{i+1}$ using Eqs. (10) and (11);

8. While the termination conditions are not met, return to step 3;

9. End loop.

\section{PROCEDURES OF MULTISCALE MORPHOLOGY ANALYSIS FOR FAULT DIAGNOSIS}

The procedure for using a multiscale morphological filter for fault diagnosis is as follows:

1. Select a multiscale $\mathrm{SE} \lambda g$, in this paper the scales are selected from 3,5 and 7 where the unit is one sampling point. (The size of the SE is subject to the length of the noise period [8])

2. For the scale of $\lambda_{i}$, the PSO is used to optimize the amplitudes of the SE; the SNR function is used as the fitness function.

3. Then, the SE with an optimized length is used to perform the multiscale morphological filter operation on the original signal and impulsive components are extracted so that the background noise can be better restrained.

4. Change the scale to $\lambda_{i+1}$, repeat steps (2 to 4 );

5. Through a weight operation the final results of the multiscale morphology analysis can be obtained:

$$
y=\sum_{i=k}^{n} \omega_{i} y_{i},
$$

where $[k, n]$ is the range of $\lambda, \omega_{i}$ is the weight of different $\lambda_{i}$. In this paper, the $\omega_{i}$ took the mean value, $\omega_{i}=1 /(n-k)$.

\section{THE VALIDATED THROUGH SIMULATION DATA}

A simulated signal is built to validate the proposed method. It is defined as:

$$
x(t)=x_{1}(t)+x_{2}(t)+x_{3}(t) .
$$

The signal $x_{1}(t)=\cos (2 \pi 30 t)+\cos (2 \pi 50 t)$ is shown in Fig.1a; the impulsive signal $x_{2}(t)$ is a typical series of exponentially decaying pulses used to simulate the impulsive signal (the repetition period is $0.0625 \mathrm{~s}$ and the impulsive function in one period is $e^{-5 t} \sin (10 \pi t)$ as shown in Fig. 1b; $x_{3}(t)$ is the Gaussian white noise with a standard deviation of 0.5 . The composite signal $x(t)$ is shown in Fig.1c.
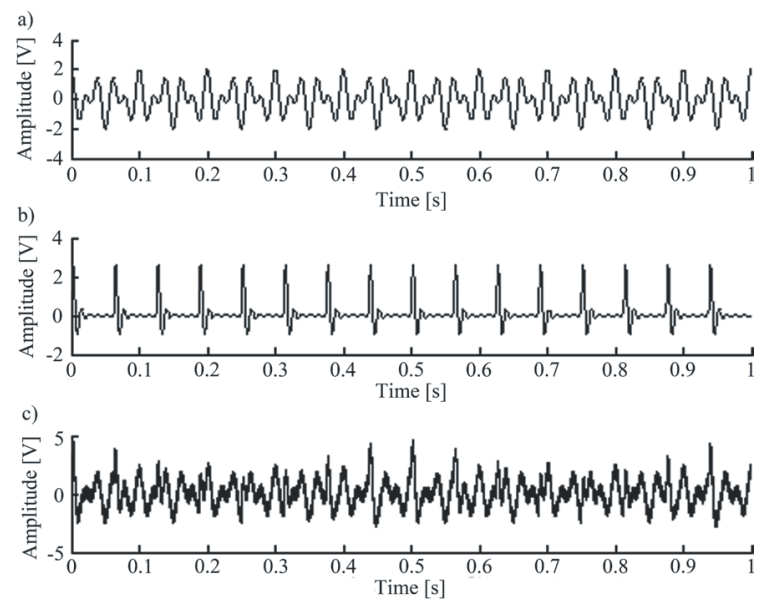

Fig. 1. The signal Waveform graph

The parameters of the PSO for optimizing the SE were set as the original position $x(t)=0$ and velocity $v(t)=0$, pbest $=0$, gbest $=0$, the population scale $m=20$, the terminal interaction time $t_{\max }=200$, the inertia weight $w=0.5, c_{1}=c_{2}=1.2$, the search space dimension $d=3$. The optimal SEs were obtained as $\{0,0.0006,0\},\{0,0.1115,0.2229,0.1115,0\}$, $\{0,0.1484,0.2968,0.4452,0.2968,0.1484\}$.

Fig. 2 is the FFT spectrum of the simulated signal, the impulsive frequency was laid in the strong harmonic frequencies (30 and $50 \mathrm{~Hz}$ ). The impulsive frequency may be covered by the noise if it is strong or the impulsive signal is weak. The purpose of the 
simulated experiment is to extract the impulsive features at 16, 32 and $48 \mathrm{~Hz}$, to suppress the harmonic features at 30 and $50 \mathrm{~Hz}$, and remove the white noise feature.

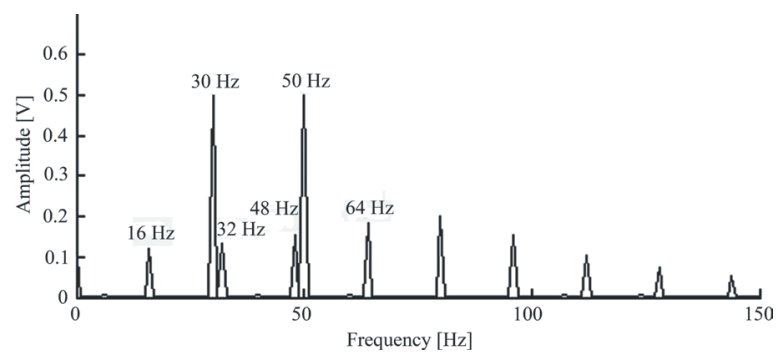

Fig. 2. The FFT spectrum of the simulated signal

The results achieved using the original singlescale morphological filter employed by Nikolaou [1] (the structure element $g=\{0,0,0\}$ ), and the method proposed in this paper are shown and compared in Fig. 3. Since the SEs were constructed according to the characters of the signal, the entire impulsive signal can be extracted from the background noise, which cannot be carried out by single-scale morphology analysis.
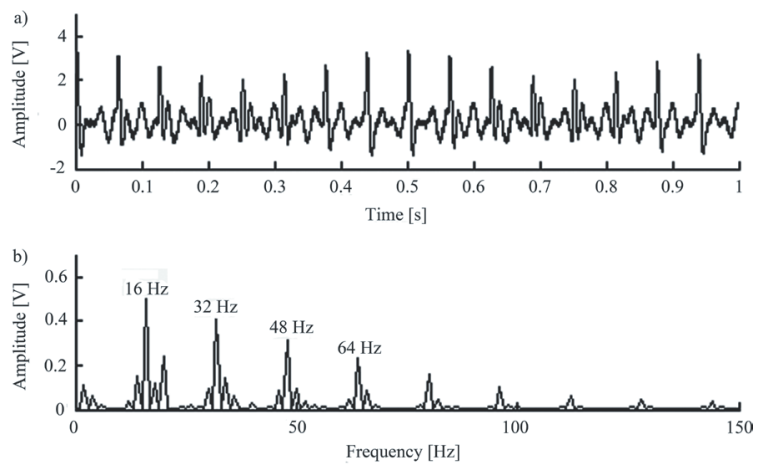

Fig. 3. The FFT spectrums of the simulated signal
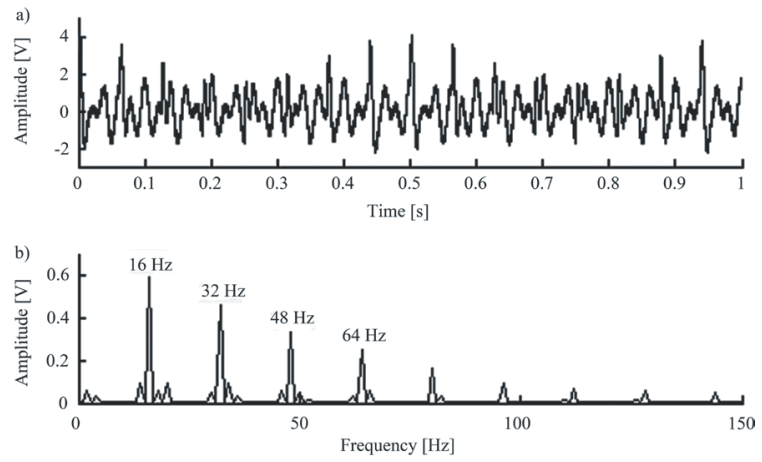

Fig. 4. The FFT spectrums of the simulated signal
The FFT spectrums of the simulated signal are shown in Figs. 3 and 4. In Fig. 3b, obvious impulsive features, i.e. 16, 32 and $48 \mathrm{~Hz}$, are presented. Compared to Fig. 4b, there are some background signals, such as at 16 and $32 \mathrm{~Hz}$, in Fig. $3 \mathrm{~b}$. This shows that the optimal multiscale morphology analysis has better performance in impulsive features extraction and noise reduction than the traditional morphology filter.

\section{EXPERIMENTAL VALIDATION}

The effectiveness of the proposed optimal multiscale morphology analysis method was evaluated using the vibration data measured in our lab. In the experimental setup detailed in Fig. 5, the power is provided by an electrical motor. To measure the vibrations three accelerometers were mounted on the square housing of the analyzed bearing.

The outer and inner races have a fault created by electro-discharge machining as shown in Figs. 6 and 7. The fault size is 0.007 inches in diameter and 0.011 inches in depth for ball, inner and outer races. The motor speed is $1721 \mathrm{RPM}(28.7 \mathrm{~Hz})$, the inner defect frequency was calculated to be $156 \mathrm{~Hz}$ and the outer defect frequency $103 \mathrm{~Hz}$.

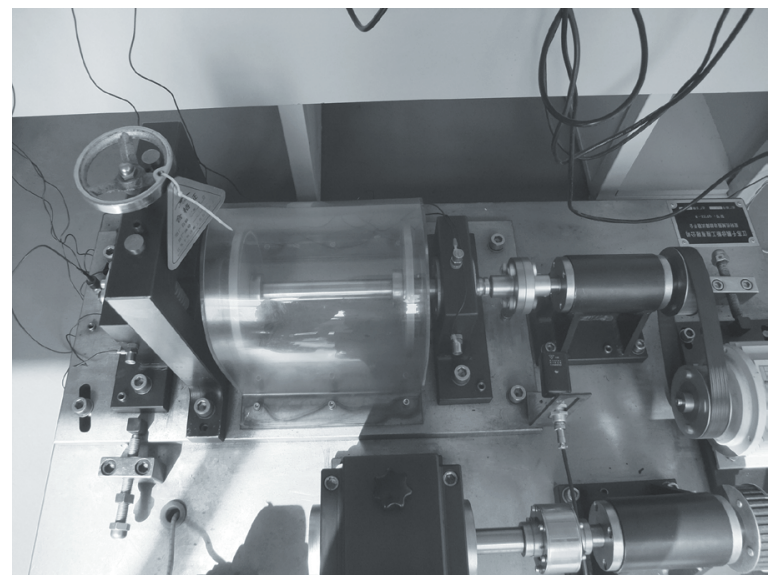

Fig. 5. Experimental setup

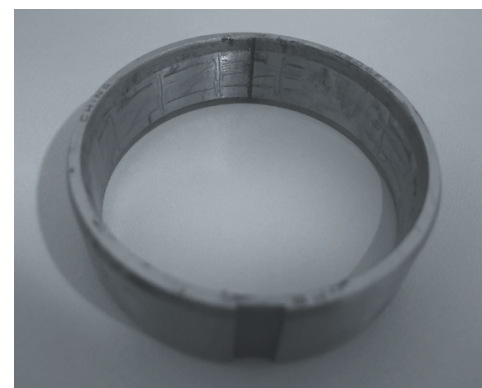

Fig. 6. Roller bearings with outer race fault 


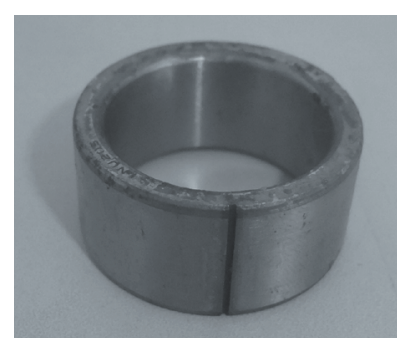

Fig. 7. Roller bearings with inner race fault

Data were collected at 12,000 samples/second, the number of sampling points is $12 \mathrm{k}$. Roller bearings with inner and outerrace faults are used for the analysis.

Their time domain waveform and FFT spectrum are shown in Figs. 8 and 10. However, due to the noise disturbance, it was difficult to obtain useful information from the analysis.
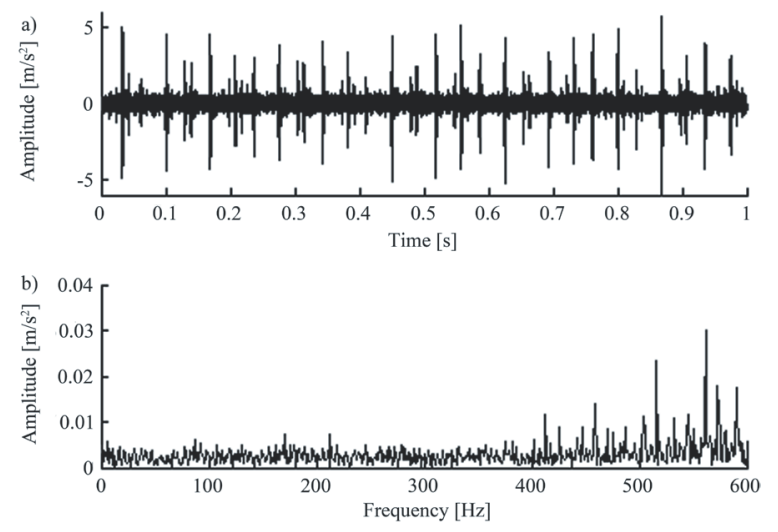

Fig. 8. Outer race fault signal waveform and FFT spectrum

For the outer race fault signal, the parameters of the PSO for optimizing the SE were set such that the original position was $x(t)=0$ and the velocity was $v(t)=0$, pbest $=0$, gbest $=0$, the population scale was $m=20$, the terminal interaction time was $t_{\max }=300$, the inertia weight was $w=0.5, c_{1}=c_{2}=$ 1.2 , and the search space dimension was $d=3$. The optimal SEs obtained were $\{0,0.001,0\},\{0,0.1003$, $0.3211,0.1003,0\},\{0,0.1156,0.2701,0.5423,0.2701$, $0.1156,0\}$.

The vibration signal was analyzed using an optimal multiscale morphology filter. Fig. 9b shows that the outer defect frequency of $103 \mathrm{~Hz}$ together with its second and third harmonics, i.e. 206 and 309 $\mathrm{Hz}$, side frequency and modulation frequency (28.7 $\mathrm{Hz}$ ) were all clearly detected. There is a good match between the expected features of the FFT spectrum and the actual fault features associated with the roller bearing with the outer race fault.
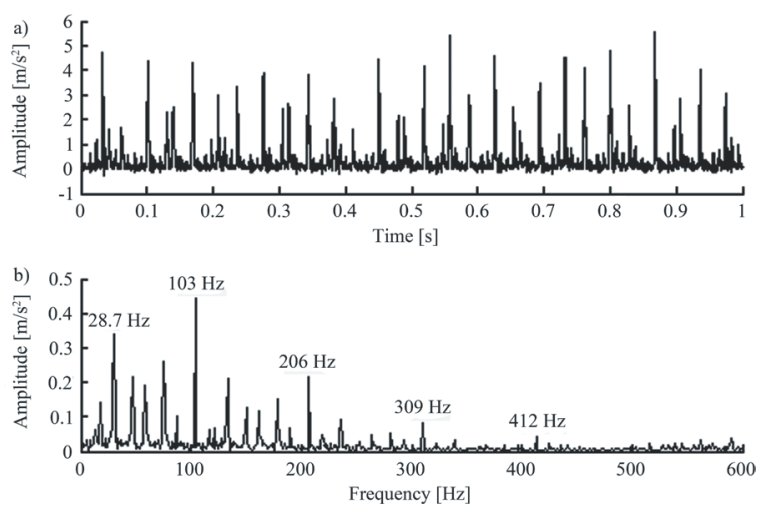

Fig. 9. Outer race fault vibration signal analyzed using an optimal multiscale morphology filter
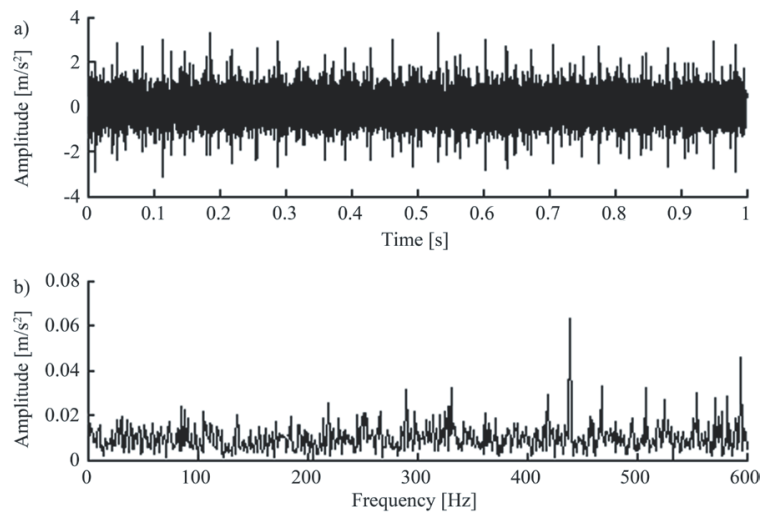

Fig. 10. Inner race fault signal waveform and FFT spectrum

For the inner race fault signal, the parameters of the PSO for optimizing the SE were set such that the original position was $x(t)=0$ and velocity was $v(t)=0$, pbest $=0$, gbest $=0$, the population scale was $m=20$, the terminal interaction time was $t_{\max }=300$, the inertia weight $w=0.5, c_{1}=c_{2}=1.2$, and the search space dimension was $d=3$. The optimal SEs obtained were $\{0,0.0006,0\},\{0,0.0703,0.1211,0.0703,0\},\{0$, $0.0156,0.1301,0.2351,0.1301,0.0156,0\}$.
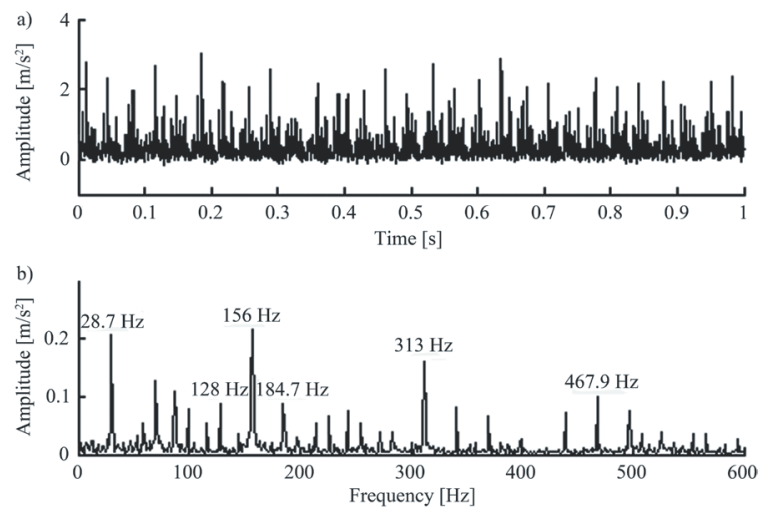

Fig. 11. Inner race fault vibration signal analyzed by optimal multiscale morphology filter 
Fig. 11b shows that the inner defect frequency $156 \mathrm{~Hz}$ together with its second and third harmonics, i.e., 313 and $467.9 \mathrm{~Hz}$, and side frequencies (156 \pm $28.7 \mathrm{~Hz})$ are prominent. The modulation frequencies $28.7 \mathrm{~Hz}$ (the frequency of rotor rotating) are also very clear. This reveals that the modified approach is effective in detecting faults in the bearing.

\section{CONCLUSIONS}

In this paper, an optimal multiscale morphology analysis based fault diagnosis approach was proposed. Compared with the traditional morphological filter, two improvements were made in the proposed method. Firstly, the morphological operation was decided with an average combination of multiscale open-closing and close-opening operation, so that not only impulses could be extracted but also the noise could be removed. Then, the structure elements were selected and optimized using aparticle swarm optimization algorithm, which has features similar to the object signal. A step-by-step procedure was also defined to illustrate how the proposed approach can be applied. The validation results show that the proposed approach is more effective and robust in extracting impulsive features than the traditional single-scale morphology analysis.

Moreover, since the SE is optimized by the PSO according to the signal, the optimized multiscale morphological filter is beneficial in improving the accuracy of mechanical fault diagnosis. However, as the redundancy increases, the complexity of the computation increases as well. This is one of the main shortcomings of the proposed transform, which should be explored in the future.

\section{ACKNOWLEDGMENT}

We would like to thank the anonymous reviewers and editors for their valuable comments and suggestions. We also thank the Science and Technology Commission of Chongqing (Grant No. cstc2012jcsfjfzhX0027), China.

\section{REFERENCES}

[1] Nikolaou, N.G., Antoniadis, I.A. (2003). Application of morphological operators as envelope extractors for impulsive-type periodic signals. Mechanical Systems and Signal Processing, vol. 17, no. 6, p. 1147-1162, DOI:10.1006/mssp.2002.1576.

[2] Su, W.S., Wang, F.T., Zhu, H. (2010). Rolling element bearing faults diagnosis based on optimal Morlet wavelet filter and autocorrelation enhancement. Mechanical Systems and Signal Processing, vol. 24, no. 5, p. 1458-1472, DOI:10.1016/j.ymssp.2009.11.011.

[3] Cheng, J.S., Yu, D.J., Yang, Y.(2006). A fault diagnosis approach for roller bearings based on EMD method and AR model. Mechanical Systems and Signal Processing, vol. 20, no. 2, p. 350-362, DOI:10.1016/j. ymssp.2004.11.002.

[4] Hu, A., Tang, G., An, L. (2006). De-noising technique for vibration signals of rotating machinery based on mathematical morphology filter. Chinese Journal of Mechanical Engineering, vol. 42, no. 4, p. 127-130, DOI:10.3901/JME.2006.04.127.

[5] Zhang, L., Yang, D., Xu, J., Chen, Z. (2007). Approach to extracting gear fault feature based on mathematical morphological filtering. Chinese Journal of Mechanical Engineering, vol. 43, no. 2, p. 71-75, DOI:10.3901/ JME.2007.02.071.

[6] Li, H., Xiao, D. (2012).Fault diagnosis using pattern classification based on one-dimensional adaptive rank-order morphological filter. Journal of Process Control, vol. 22, no. 2, p. 436-449, DOI:10.1016/j. jprocont.2011.12.005.

[7] Maragos, P. (1989). Pattern spectrum and multiscale shape representation. IEEE Transactions on Pattern Analysis and Machine Intelligence, vol. 11, no. 7, p. 701-716, DOI:10.1109/34.192465.

[8] Ji, T.Y., Lu, Z., Wu, Q.H. (2007). Optimal soft morphological filter for periodic noise removal using a particle swarm optimiser with passive congregation. Signal Processing, vol. 87, no. 11, p. 2799-2809. DOI:10.1016/j.sigpro.2007.05.024.

[9] Zhang, Z.Y., Zhang, H.Y. (2004). Principal manifolds and nonlinear dimensionality reduction via tangent space alignment. Journal of Shanghai University (English Edition), vol. 8, no. 4, p. 406-424, DOI:10.1007/s11741-004-0051-1 